\title{
Utilization of chronic lung disease treatment before the respiratory syncytial virus season as palivizumab prophylaxis qualifier in the American Academy of Pediatrics Guidelines
}

\author{
Yoonyoung Choi ${ }^{1}$ - H. Cody Meissner ${ }^{2,3} \cdot$ Christian Hampp $^{4} \cdot$ Haesuk Park ${ }^{1,5} \cdot$ Almut G. Winterstein ${ }^{1,5,6}$
}

Received: 10 March 2021 / Revised: 31 July 2021 / Accepted: 2 August 2021 / Published online: 7 August 2021

( $)$ The Author(s), under exclusive licence to Springer-Verlag GmbH Germany, part of Springer Nature 2021

\begin{abstract}
Guidelines from the American Academy of Pediatrics recommend palivizumab immunoprophylaxis for children with CLD in their second year of life if they continue to need treatment within 6 months before the RSV season. The utilization patterns of treatment (chronic corticosteroid therapy, diuretic therapy, or supplemental oxygen) are not well understood. We examined variations in CLD treatment for ten consecutive 20-day segments preceding RSV season onset. Among infants and children with CLD $(n=19,026), 35.2 \%$ received one or more medical treatments for CLD any time within 200 days before entering the second RSV season: 8.6\%, 3.2\%, and 29.7\% received supplemental oxygen, diuretics, and corticosteroids, respectively. Utilization decreased as infants' age increased with corticosteroids surpassing oxygen and diuretics. To avoid the capture of intermittent use of corticosteroids for acute infections, we found requiring a minimum of 45 days cumulative exposure was reasonable to determine chronic use.
\end{abstract}

\section{What is Known:}

- Guidelines from the American Academy of Pediatrics recommend palivizumab immunoprophylaxis for children with CLD in their second year of life if they continue to need treatment within 6 months before the RSV season.

- The utilization patterns of treatment (chronic corticosteroid therapy, diuretic therapy, or supplemental oxygen) are not well understood. A definition of chronic corticosteroid therapy in this setting is not available.

\section{What is New:}

- Among infants and children with CLD of prematurity, 35.2\% received one or more medical treatments for CLD any time within 200 days before entering the second RSV season: $8.6 \%, 3.2 \%$, and $29.7 \%$ received oxygen, diuretics, and corticosteroids, respectively. Utilization decreased as infants' age increased with corticosteroids surpassing oxygen and diuretics.

- A minimum of 45 days cumulative corticosteroid use within the past 90 days would accurately capture chronic use to fulfill criteria for immunoprophylaxis while limiting the inclusion of intermittent use of corticosteroids for acute infections.

Communicated by Nicole Ritz

Almut G. Winterstein

Almut@ufl.edu

Yoonyoung Choi

chyoonyo@ufl.edu

H. Cody Meissner

cmeissner@tuftsmedicalcenter.org

Christian Hampp

Christian.Hampp@fda.hhs.gov

Haesuk Park

HPark@cop.ufl.edu

1 Department of Pharmaceutical Outcomes \& Policy, College of Pharmacy, University of Florida, 1225 Center Drive,

Gainesville, FL 32611, USA
2 Tufts Children's Hospital, Tufts Medical Center, Boston, USA

3 Tufts University School of Medicine, Boston, USA

4 Office of Surveillance and Epidemiology, Center for Drug Evaluation and Research, Food and Drug Administration, Silver Spring, USA

5 Center for Drug Evaluation and Safety (CoDES), University of Florida, 1225 Center Drive, Gainesville, FL 32611, USA

6 Department of Epidemiology, College of Public Health and Health Professionals and College of Medicine, University of Florida, 1225 Center Drive, Gainesville, FL 32611, USA 
Keywords Chronic lung disease $\cdot$ Treatment utilization pattern $\cdot$ Respiratory syncytial virus $\cdot$ Palivizumab immunoprophylaxis

\section{Abbreviations}

CLD Chronic lung disease of prematurity

RSV Respiratory syncytial virus

RTI Respiratory tract infection

\section{Introduction}

Guidelines from the American Academy of Pediatrics recommend palivizumab immunoprophylaxis for children with chronic lung disease of prematurity (CLD) in the second year of life if they require medical treatment (any of chronic corticosteroid therapy, diuretic therapy, or supplemental oxygen) within 6 months before the onset of the respiratory syncytial virus (RSV) season [1]. This requirement is used to identify children with severe lung disease who are most likely to require hospitalization due to RSV infection and thus most likely to benefit from immunoprophylaxis. Limited information is available regarding the frequency and duration of medical treatment in this clinical scenario. Thus, to inform clinical decision-making, we examined the utilization patterns of medical treatment in this cohort.

\section{Methods}

We conducted a retrospective cohort analysis using the Medicaid Analytic eXtract (MAX) databases and Birth Certificate data from Florida and Texas [2, 3] 1999-2010 during which a unique linkage of data was completed. Texas and Florida have the 2nd and 4th highest number of annual births in the United States (U.S.) [4]; and Medicaid covers healthcare for more than 4 of 10 births across the U.S [5, 6]. Children with records in both datasets had at least one medical encounter with a diagnosis of CLD (International Classification of Diseases, 9th revision, Clinical Modification code [ICD9-CM] 770.7: chronic respiratory disease arising in the perinatal period, or 769: respiratory distress syndrome (RDS) in newborn, which has been previously validated [7]). Subjects were included if they were born at $<32$ weeks gestation based on vital records; had $\geq 200$ days of continuous enrollment in Medicaid; were $\leq 24$ months of age at the start of the RSV season; and had received supplemental oxygen or mechanical ventilation during the first 90 days after birth (as ascertained from vital records and claims data). Details of operational definitions have been published in a previous study [8].
We defined medical treatment of CLD as the continued administration of diuretic therapy, supplemental oxygen, or corticosteroid therapy. Diuretic and corticosteroid therapies were ascertained from pharmacy claims using American Hospital Formulary Service (AHFS) class 40:28 diuretics and AHFS 24:32.20 mineralocorticoid (aldosterone) receptor antagonists, and 68:04 adrenals, respectively. We calculated the duration of treatment based on pharmacy-entered days' supply (for corticosteroids and diuretics), assuming that treatment would start on the day of dispensing. Oxygen supplementation or ventilation was ascertained from medical claims using Healthcare Common Procedure Coding System codes (E0424, E0425, E0430, E0431, E0433, E0434, E0435, E0439, E0440, E0441, E0442, E0443, E0444, E0450, E1390, E1391, E1392, E1400, E1401, E1402, E1403, E1404, E1405, E1406), and ICD-9-CM diagnosis (V462) and procedure (93.96) codes, and from documentation of the need for assisted ventilation on birth certificates. We calculated the duration of supplemental oxygen requirement based on recorded length of stay (for inpatient claims) and commonly observed intervals between claims (for outpatient claims, 30 days or 1 day when attributed to procedures or diagnoses, respectively).

To operationalize "chronic" corticosteroid therapy, we reviewed dispensing patterns among children with CLD diagnoses during 0-24 months of age. Most corticosteroid prescription refills (81\%) occurred within 90 days of the previous fill and included both inhaled and oral dosage forms. Inhaled dosage forms were commonly dispensed for a long days' supply. Accordingly, we calculated cumulative days' supply including both oral and inhaled corticosteroids by looking back 90 days from an index corticosteroid dispensing. For example, for a corticosteroid fill on March 31st 2010, every corticosteroid fill between January 1st and March 31st would contribute to determining the number of cumulative days' supply during the 90-day look-back period. Each corticosteroid dispensing served as an anchor to calculate previous corticosteroid exposure. We evaluated four thresholds to define "chronic" corticosteroid therapy: any corticosteroid dispensing, or $\geq 30$-, 45-, or 60-day cumulative days' supply during the past 90 days.

We also examined the concurrence of RTI infections to help to differentiate intermittent versus chronic corticosteroid therapy. Utilization prevalence rates were calculated in 20-day segments across 200 days before the onset of the RSV season. As done in a previous study [8], we defined RSV season onset as the first of the 26 consecutive weeks with the highest incidence of RSV hospitalizations in Florida and Texas, accounting for palivizumab exposure. We further stratified utilization patterns for infants and 
children by chronologic age: age $200-365$ days and age 366-730 days at the start of the RSV season.

This study was approved by the institutional review and privacy boards of the University of Florida, the Centers for Medicare and Medicaid Services, and the Florida and Texas Departments of Health.

\section{Results}

Among infants and children with CLD $(n=19,026), 35.2 \%$ received one or more ongoing medical treatments for CLD any time within 200 days before entering their second RSV season: $8.6 \%, 3.2 \%$, and $29.7 \%$ received oxygen, diuretics, and corticosteroids, respectively. Among children aged 200-365 days at the start of the RSV season, the most distant time of the 200-day look-back period relied on oxygen and to a lesser extent, diuretic therapy (Fig. 1). In the second year of life, chronic corticosteroid use surpassed both diuretics and oxygen.

Utilization patterns of oxygen and diuretics within 200 days prior to the RSV season were not affected by RTI trends (Fig. 1). Prevalence rates of oxygen and diuretics use decreased as infants became older, approaching the start of the RSV season. In contrast, corticosteroid dispensing showed a bimodal distribution with an unexpected increase at the beginning of the season (i.e., when children had the highest age and would be expected to have decreased CLD severity).

The increase was consistent with RTI incidence patterns in the study populations: the 200-day look-back period overlapped with an offset of the previous cold season and an onset of the current cold season, likely creating a composite of corticosteroid therapy that was used for seasonal acute respiratory tract infections as well as unresolved CLD. To associate corticosteroid use with the underlying CLD severity, rather than a seasonal respiratory infection, we compared trends of our various definitions of chronic corticosteroid based on cumulative days' supply during the past 90 days. As a result, shorter courses of chronic corticosteroid use paralleled the increasing RTI infection rates at RSV season onset, but this effect was attenuated when requiring $\geq 45$ cumulative days' supply to define chronic steroid use.
Fig. 1 Prevalence of CLD treatment and RTI before the start of RSV season. CLD infants between 200 and 365 days of age at the start of the season $(n=6980)$ and CLD children between 366 and 730 days of age at the start of the season $(n=12,046)$. Data source: 12 years of data from MAX databases (1999-2010) linked to Florida and Texas Birth Certificate data

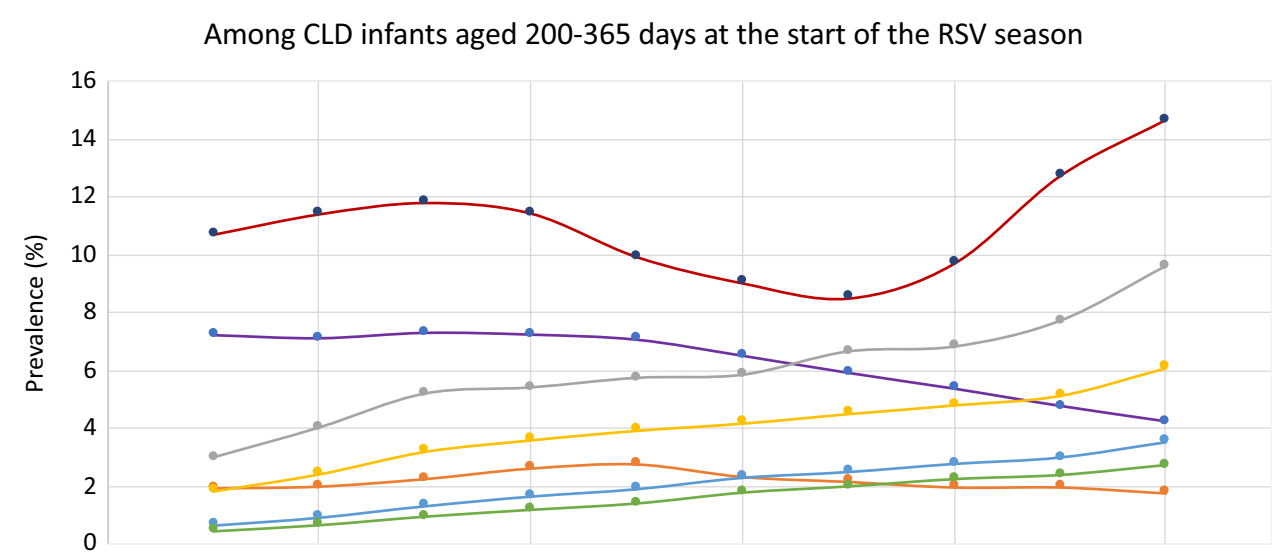

Among CLD children aged 366-730 days at the start of the RSV season

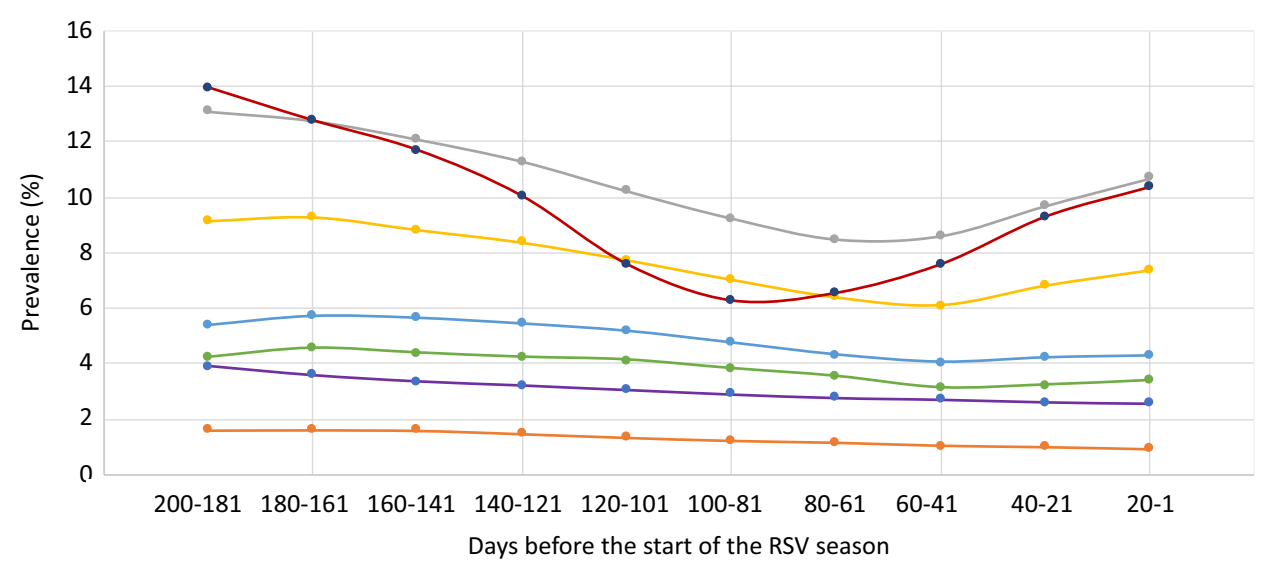

$\rightarrow$ Oxygen $\rightarrow$ Diuretics $\rightarrow$ Steroids $($ all $) \rightarrow$ Steroids $(30 d) \rightarrow$ Steroids $(45$ d) $\rightarrow$ Steroids $(60 d) \rightarrow$ RTI 


\section{Discussion}

We described patterns of medical treatment among children diagnosed with CLD who are entering their second RSV season. As expected, we observed decreasing utilization trends in oxygen and diuretics with increasing chronologic age. In the absence of a widely acknowledged definition of chronic corticosteroid therapy in this setting, our multiple threshold analyses elucidated the heterogeneous purpose of corticosteroids use, given their role in inflammation reduction [9]. Our analysis suggests that the use of a more restrictive threshold (e.g., $>45$-day cumulative supply within the past 90 days) for corticosteroid prescriptions would more accurately capture chronic use to fulfill criteria for immunoprophylaxis.

A few limitations of this study are noteworthy. While Medicaid billing records are a reliable source for ascertaining medical support for CLD [10], claims-based treatment duration information can be inaccurate. Second, our CLD cohort may not exactly mirror those recommended for palivizumab immunoprophylaxis in guidelines. For example, our data could not provide the actual duration of oxygen use that guidelines require to determine the eligibility (i.e., supplemental oxygen at least 28 days after birth). Third, our operational definition for chronic corticosteroid use included inhaled corticosteroids based on an empirical finding of its prevalent use. Given a continuous evaluation of the beneficial effects of inhaled corticosteroid on the pulmonary system [11], we acknowledge a restriction to systemic corticosteroids, which were not investigated in this study on their own, could have been relevant. Finally, our study findings may not be generalizable to other countries where guidelines for CLD treatment can be different from those in the U.S.

Our findings on the utilization of CLD treatment could inform guidelines to specify treatment requirements as a proxy for CLD severity (e.g., supplemental oxygen, diuretics, or $\geq 45$ cumulative days' supply of corticosteroids) and clarify the size of the eligible population for palivizumab prophylaxis among those in the second year of life. Recently, the unprecedented COVID-19 pandemic has changed RSV epidemiology, causing unexpected variations in the RSV season onset and offset [12]. In the continuing presence of a coronavirus pandemic, revised guidance may be required to determine the season onset to consider palivizumab prophylaxis and evaluate the continuous needs of CLD treatment.

\section{Conclusion}

Approximately one-third of infants and children with CLD received one or more medical treatments for CLD any time within 200 days before entering the second RSV season.
Utilization of oxygen and diuretics decreased as infants' age increased, surpassed by corticosteroids. To define chronic corticosteroid therapy as the criteria for immunoprophylaxis, we suggest considering at least 45 days of cumulative use within the past three months.

Authors' Contributions Dr. Choi conceptualized and designed the study, carried out the data analyses, interpreted the data, drafted the initial manuscript, and reviewed and revised the manuscript.

Dr. Meissner conceptualized and designed the study, substantially contributed interpretation of data, and reviewed and revised the manuscript.

Dr. Hampp substantially contributed interpretation of data, and reviewed and revised the manuscript.

Dr. Park substantially contributed interpretation of data, and reviewed and revised the manuscript.

Dr. Winterstein conceptualized and designed the study, acquired data, supervised the data analyses, substantially contributed interpretation of data, and reviewed and revised the manuscript.

All authors approved the final manuscript as submitted and agreed to be accountable for all aspects of the work.

\section{Declarations}

Ethics approval This study was approved by the institutional review and privacy boards of the University of Florida, the Centers for Medicare and Medicaid Services, and the Florida and Texas Departments of Health. We thank the Florida Department of Health and The Texas Department of State Health Services and the Health and Human Services Commission for provision of the vital record data. Any published findings and conclusions are those of the authors and do not necessarily represent the official position of the Florida Department of Health.

Consent for publication All authors approved the final manuscript as submitted and agreed to be accountable for all aspects of the work.

Conflict of interest The authors have no conflicts of interest relevant to this article to disclose. Dr. Choi participated in the study while working at the University of Florida. Dr. Choi's current affiliation is Merck \& Co, Inc., Center for Observational and Real-World Evidence (CORE). The views and opinions expressed in this article do not represent the views and opinions of any public or private entity (e.g., agency, government, organization, institution, or company). This article reflects the views of the authors and should not be construed to represent the views or policies of the Food and Drug Administration, the Florida Department of Health, or the Texas Department of State Health Services.

\section{References}

1. Committee on Infectious Diseases (2014) Updated guidance for palivizumab prophylaxis among infants and young children at increased risk of hospitalization for respiratory syncytial virus infection. Pediatrics 134(2):e620-638

2. Florida Department of Health (2015) Certificates-birth, death, fetal death, marriage and divorce. Available at https://www.dshs. texas.gov/vs/. Accessed 1 July 2015

3. Texas Health and Human Services (2015) Texas vital statistics. Available at http://www.floridahealth.gov/certificates/certificates/. Accessed 1 July 2015 
4. National Center for Health Statistics (2021) Birth Data. Available at https://www.cdc.gov/nchs/fastats/state-and-territorial-data.htm. Accessed 29 July 2021

5. The Kaiser Family Foundation (2007) Medicaid's role in family planning. Available at https://www.guttmacher.org/sites/default/ files/pdfs/pubs/IB_medicaidFP.pdf. Accessed 15 Mar 2017

6. Sonfield A, Kost K, Gold RB, Finer LB (2011) The public costs of births resulting from unintended pregnancies: national and statelevel estimates. Perspect Sex Reprod Health 43(2):94-102

7. Landry JS, Croitoru D, Menzies D (2012) Validation of ICD-9 diagnostic codes for bronchopulmonary dysplasia in Quebec's provincial health care databases. Chronic Dis Inj Can 33(1):47-52

8. Choi Y, Meissner HC, Hampp C, Park H, Brumback B, Winterstein AG (2020) Calibration of chronic lung disease severity as a risk factor for respiratory syncytial virus hospitalization. J Ped Infect Dis Soc 10(3):317-25
9. Baveja R, Christou H (2006) Pharmacological strategies in the prevention and management of bronchopulmonary dysplasia. Semin Perinatol 30(4):209-218

10. Crystal S, Akincigil A, Bilder S, Walkup JT (2007) Studying prescription drug use and outcomes with medicaid claims data: strengths, limitations, and strategies. Med Care 45(10 Supl 2):S58-65

11. Shinwell ES, Portnov I, Meerpohl JJ, Karen T, Bassler D (2016) Inhaled corticosteroids for bronchopulmonary dysplasia: a metaanalysis. Pediatrics 1;138(6):e20162511

12. Olsen SJ, Winn AK, Budd AP et al (2020-2021) Changes in influenza and other respiratory virus activity during the Covid19 pandemic-United States. MMWR 70(29):1013-1019

Publisher's Note Springer Nature remains neutral with regard to jurisdictional claims in published maps and institutional affiliations. 